\title{
The role of Cyp19a1 in female pathway of a freshwater turtle (Mauremys reevesii) with temperature-dependent sex determination
}

$1 \quad$ Peng-fei Wu ${ }^{1,3}$, Xi-feng Wang ${ }^{*}$, Fei Gao², Wei-guo Du ${ }^{1}$

$2 \quad{ }^{1}$ Key Laboratory of Animal Ecology and Conservation Biology, Institute of Zoology, Chinese

3 Academy of Sciences, Beijing, 100101, People's Republic of China

$4 \quad{ }^{2}$ State Key Laboratory of Stem Cell and Reproductive Biology, Institute of Zoology, Chinese

5 Academy of Science, Beijing, 100101, People's Republic of China

$6 \quad{ }^{3}$ University of Chinese Academy of Sciences, Beijing, 100049, People's Republic of China

7 * Correspondence:

$8 \quad$ Xi-feng Wang

9 wangxifeng@ioz.ac.cn

10 Keywords: Temperature-dependent sex determination1, Sex-determining gene2, Cyp19a13, 11 Ovarian differentiation4, Mauremys reevesii5.

\section{Abstract}

13 The molecular mechanism of temperature-dependent sex determination (TSD) in reptiles has been drawn great interest from biologists for several decades. However, which genetic factors are essential for TSD remain elusive, especially for the female sex determination process. Cyp 19a1, encodes an enzyme of aromatase catalyzing the conversion of testosterone to estrogen, has been confirmed to modulate steroid hormones involved in the sexual differentiation of many species, but whether it has a critical role in determining the gonadal sexual fate in TSD is still to be elucidated. Here, we identified that Cyp19al expression exhibited a temperature-dependent, sexually dimorphic expression pattern, preceding gonadal sex differentiation in a TSD turtle Mauremys reevesii. Cyp19al expression in gonads increased dramatically when embryos developed at high femaleproducing temperatures (FPT), but were extremely low throughout embryogenesis at low maleproducing temperatures (MPT). Cyp19al expression increased rapidly in response to the temperature shift from MPT to FPT in developing gonads. The sexual phenotype of turtles was successfully reversed by aromatase inhibitor treatment at FPT, and by estrogen treatment at MPT, accompanied with the rapid upregulation of Cyp19a1. These results demonstrate that Cyp19al is essential for the female sex determination process in M. reevesii, indicating its vital role in the female pathway of TSD.

\section{Introduction}

Two sexes universally exist in many animals, and a diverse of mechanisms that determine sex have evolved across different lineages. In vertebrates, sex determination mainly divided into two types: genotype sex determination (GSD) and environmental-dependent sex determination (ESD) (Valenzuela and Lance, 2004). Temperature-dependent sex determination (TSD) is a notable ESD existing in some reptiles, in which the incubation temperature of developing embryos determines the gonadal sex (Charnier, 1966; Ferguson and Joanen, 1982; Pieau et al., 1999). 
In reptiles, the molecular mechanisms underlying TSD have undergone several decades of research. Early studies mainly focused on hormone-dependent mechanisms, and clone and/or expression of some classical sex-related genes involved in GSD system (e.g., Wt1; Dmrt1; Amh) in TSD species (Gutzke and Bull, 1986; Wibbels and Crews, 1992; Spotila and Hall, 1998; Pieau et al., 1999; Gabriel et al., 2001). Later studies demonstrated that several of those genes exhibited temperaturedependent expression patterns during thermo-sensitive period (TSP), prior to gonadal sex differentiation (Shoemaker et al., 2007a; Shoemaker-Daly et al., 2010; Matsumoto and Crews, 2012). Recently, transcriptomes of TSD taxa identified a great number of candidate sex-determining genes in TSD reptiles (Czerwinski et al., 2016; Yatsu et al., 2016; Radhakrishnan et al., 2017). In addition, genetic manipulation techniques (e.g., RNA interference) were introduced to identify the function of sex-related genes in sex determination of reptiles (Shoemaker-Daly et al., 2010; Sifuentes-Romero et al., 2013; Ge et al., 2017). For example, Dmrtl determines the male fate in a TSD turtle Trachemys scripta through the Temperature-Ca2+-pSTAT3-Kdm6b-Dmrt1 pathway, revealing a direct genetic link between epigenetic mechanism and TSD (Ge et al., 2017; Ge et al., 2018; Weber et al., 2020). Despite several decades of research on TSD mechanisms in reptiles, the role and function of key sexrelated genes in female sex determination process remains unverified using robust molecular evidences including gene expression and genetic manipulation.

Cyp19a1 (Cytochrome P450 Family 19 Subfamily A Member 1) encodes an endoplasmic reticulum enzyme called aromatase, catalyzing the conversion of testosterone to estrogen (Simpson et al., 1994), which is critical for estrogen synthesis. Estrogen plays a critical role in the sexually dimorphic development of anatomical, functional and behavioral characteristics that are essential for female development (Nelson and Habibi, 2013; Hamilton et al., 2014; Tokunaga et al., 2014). Cyp19al has been identified to modulate steroid hormones involving in the sexual differentiation of GSD species (Elbrecht and Smith, 1992; Crews and Bergeron, 1994; Kitano et al., 2000; Sakata et al., 2005). In GSD vertebrates like medaka (Oryzias latipes) and chicken (Gallus gallus), Cyp19al generally exhibits a sexually dimorphic expression pattern in female gonads at early embryonic days and robustly expresses within the cytoplasm in ovarian medullas; the gain- and loss-of-function analyses further provide strong evidence that Cyp19al is both necessary and sufficient for ovarian differentiation (Lambeth et al., 2013; Mawaribuchi et al., 2014; Nakamoto et al., 2018; Jin et al., 2020). Although Cyp 19al is not required for the fetal sexual differentiation in mouse (Mus musculus), it is essential for granulosa cell phenotype maintaining and follicle formation in postnatal mammalian ovaries (ERICKSO et al., 1979; Britt et al., 2001; Meseke et al., 2018).

Given its vital role in GSD system, Cyp19al has long been deemed to be involved in the female pathway of TSD (Ramsey et al., 2007; Fernandino et al., 2008; Parrott et al., 2014; Tang et al., 2017). Previous studies demonstrated that Cyp19al transcripts were detected in adrenal-kidney-gonad complexes (AKGs) after the TSP of sex determination in the American alligator (Alligator mississippiensis) and several TSD turtles, denying its role as an initial trigger for sex determination (Gabriel et al., 2001; Murdock et al., 2003; Valenzuela and Shikano, 2007). In contrast, recent transcriptomic data showed that Cyp19al exhibited a female-specific expression pattern in early embryonic gonads during the late TSP (embryonic developmental stage 19) in several TSD turtles (Czerwinski et al., 2016; Radhakrishnan et al., 2017). Therefore, the role of Cyp19al in TSD remains unsolved in reptiles and may differ among species. Moreover, these previous studies merely provide the mRNA expression of Cyp19al in embryonic gonads or even AKGs, which is not enough for making an unequivocal conclusion of Cyp19al function. Further studies involving multi-level evidences (e.g., gene expression pattern, gene thermo-sensitivity, hormone/genetic manipulation analysis) in embryonic gonads are needed to identify the function of this gene. 
82

83

84

85

86

87

88

89

90

91

92

93

94

95

96

97

98

99

100

101

102

103

104

105

106

107

108

109

110

111

112

113

114

115

116

117

118

119

120

121

122

In our previous study, we have identified that Cyp $19 a 1$ exhibited a differential expression pattern in the AKGs between male-producing temperature (MPT) and female-producing temperature (FPT) in the freshwater turtle Mauremys reevesii, a TSD species (Tang et al., 2017). In this study, we firstly identified the expression pattern of Cyp19al by sampled embryonic gonads at different developmental embryonic stages at FPT and MPT, and further examined the thermo-sensitivity of Cyp19al via temperature shifts incubation experiments in this turtle species. To verify the role of Cyp19al in inducing female differentiation, we administered estrogen to MPT embryos to induce male-to-female sex reversal and detected the Cyp19al expression. Furthermore, we examined the necessity of Cyp19al in female determination process through handling aromatase inhibitor to FPT embryos. Collectively, our findings revealed that Cyp19al played a vital role in the female sex determination pathway of a TSD turtle, which provided new insight into our understanding of female sex-determining pathways in TSD reptiles.

\section{RESULTS}

\subsection{Characterization of Cyp19a1 gene in M. reevesii.}

In M. reevesii, the complete cDNA sequence of Cyp19a1 was 1995 base pairs (bp), with a 126 bp 5' untranslated region (UTR), a 355 bp 3' UTR and an open reading frame (ORF) of 1515 bp that encoded a protein of 504 amino acids (Fig. S1A). The deduced amino acid sequence of $M$. reevesii Cyp $19 a 1$ shared $84.9 \%, 84.4 \%, 75.1 \%$, and $53.5 \%$ identity with that of chicken, lizard, human, and zebrafish, respectively (Fig. S1B), indicating that $M$. reevesii Cyp19al was evolutionary more closely related to chicken, lizard and human than to fish (Fig. S1C).

Cyp19a1 mRNA was abundantly expressed in ovary, but not in testis of adult turtles (Fig. S2A). The adult testis had a dense medulla with seminiferous cords, while ovary showed a developing follicular system (Fig. S2B).

\subsection{Sexually dimorphic expression of $\mathrm{Cyp} 19 \mathrm{al}$ in early embryonic gonads of $\mathrm{M}$. reevesii.}

The Cyp19a1 transcripts started to express in FPT gonads at embryonic developmental stage of 16, which occurred at early TSP, and clearly prior to gonadal sexual differentiation. Cyp19al expression increased dramatically as embryos developed: the transcripts of Cyp 19al in FPT gonads was only dozens of times of MPT gonads at developmental stage of 16, but was thousands of times or even higher at developmental stage of 19 or 25 . In contrast, MPT gonads exhibited extremely low expression of Cyp19al throughout embryogenesis (Fig. 1).

\subsection{Thermo-sensitivity of Cyp19a1 in M. reevesii.}

The expression level of Cyp19al in turtle gonads obviously decreased with reducing temperatures from $31^{\circ} \mathrm{C}$ to $27^{\circ} \mathrm{C}$ at both developmental stage of 18 (during TSP) and 21 (after TSP) (Fig. 2A, B). In addition, Cyp19al expression responded rapidly to the temperature shift in developing gonads when embryos were transferred from MPT to FPT at stage 16. Cyp19al increased slightly above the MPT-typical level at early stage 17 and reached significantly high levels at the subsequent stages. Interestingly, Cyp19al expression showed a time lag in response to the temperature shift in gonads transferred from FPT to MPT, continuously upregulating until stage 19 and then decreasing to the MPT-typical level (Fig. 2C).

\subsection{Upregulation of Cyp19a1 in feminized MPT gonads during male-to-female sex reversal in} M. reevesii. 
In MPT embryos with estrogen treatment, the gonads exhibited female-like morphology, with a degenerated medulla and a thickened outer cortex at stage 25 (Fig. 3A-F'). Meanwhile, a significant upregulation in Foxl2 and Rspol, and downregulation of Dmrtl and Sox9 were detected in feminized MPT embryos at stage 25 (Fig. 3G). Sox9 protein was highly expressed in the medulla of MPT gonads, but decreased dramatically in feminized MPT gonads induced by estrogen at either developmental stage of 21 or 25 (Fig. 3H-J', Fig. S3A-C',). Correspondingly, germ cells in MPT gonads with estrogen treatments mainly enriched in the developed outer cortex with few germ cells localized in the medulla (Fig. 3L-L', Fig. S3E-E'), exhibiting a female-like distribution pattern (cortical localization of germ cells) rather than a male pattern (medullary cord distribution of germ cells) (Fig. 3K-K', M-M', Fig. S3D-D', F-F'). Moreover, Cyp19al transcripts responded rapidly to estrogen treatment and were significantly upregulated from stage 17 onwards (Fig. 3N).

\subsection{Masculinization of FPT embryos following aromatase inhibitor treatment in M. reevesii.}

In FPT embryos treated with aromatase inhibitor, gonads became shortened and vascularized, exhibiting male-like morphology, characterized by a dense medulla with a number of primordial germ cells and a degenerated cortex (Fig. 4A-E). Sox9 protein expressed specifically in the nuclei of precursor Sertoli cells in MPT gonads, consistent with the FPT gonads treated by aromatase inhibitor (Fig. 4G-I', Fig. S4A-C'), The distribution of vasa-positive germ cells in FPT gonads following aromatase inhibitor treatment displayed a male-like medulla localization pattern (Fig. 4J-L', , Fig. S4D-F').

\section{DISCUSSION}

143 Cyp19al was thought to play an important role in the TSD female pathway, but it lacks functional 144 analysis. Supporting this long-standing assumption of Cyp19al function, we found that Cyp19al expression showed a temperature-dependent, sexually dimorphic expression pattern prior to gonadal sexual differentiation (Fig.1), and was capable of responding rapidly to incubation temperature shift (Fig.2). In addition, Cyp19al expression rapidly upregulated in sex-reversed gonads at MPT by estrogen treatment (Fig.3), and aromatase inhibitor induced masculinization of FPT embryos (Fig.4). These results indicated that Cyp19al could determine the fate of the bipotential gonad in a TSD turtle Mauremys reevesii, and likely played a critical role in female determination pathway of TSD reptiles.

In previous studies, sex-related gene expression studies of TSD reptiles mainly based on two types of methods: qRT-PCR by individuals' AKGs (inaccurate sampling) (Western et al., 2000; Gabriel et al., 2001; Valenzuela and Shikano, 2007; Tang et al., 2017), and transcriptomes by individuals' gonads (lack of identification) (Czerwinski et al., 2016; Yatsu et al., 2016; Radhakrishnan et al., 2017). These studies demonstrated that Cyp19al gene exhibited a female-specific pattern during the end of TSP or even after TSP (Gabriel et al., 2001; Valenzuela and Shikano, 2007; Czerwinski et al., 2016; Tang et al., 2017), which indicated its vital role in sexual differentiation process but denied its role as an initial trigger in TSD. For example, Cyp19al transcripts merely expressed in gonads or AKGs during the late TSP (stage 19) in Trachemys scripta and Chrysemys picta (Radhakrishnan et al., 2017), or after the TSP in Alligator mississippiensis (Gabriel et al., 2001; Czerwinski et al., 2016). Similarly, in $M$. reevesii, early study reported that Cyp19al expression in FPT AKGs was slightly above the MPT AKGs after stage 17 (Tang et al., 2017). However, AKGs could mask earlier gonadal differential expression of sex-related genes in TSD turtles (Shoemaker et al., 2007b; Valenzuela et al., 2013). To remove this potential confounding, we identified the gene expression in $M$. reevesii gonads in the present study. Interestingly, we found that the FPT-specific expression of Cyp19al transcripts were detected as early as stage 16 by qRT-PCR, revealing the early female-specific 
expression pattern of Cyp 19al that precedes gonadal morphological differentiation (Fig. 1). These results strongly imply that Cyp19al is an early regulator for female determination in TSD turtles and involved in the early TSD female pathway but not just sexual differentiation.

170 Cyp19al has been previously showed temperature dependency in TSD reptiles (Gabriel et al., 2001; 171 Murdock et al., 2003; Radhakrishnan et al., 2017). In TSD turtles, the gonad Cyp19al transcripts 172 were highly expressed when incubated in $32^{\circ} \mathrm{C}$, and maintained at a low level in $26^{\circ} \mathrm{C}$ throughout embryonic development (Czerwinski et al., 2016; Tang et al., 2017). These studies showed a sexually dimorphic expression of Cyp19al, but the details of the Cyp19al expression influenced by temperature has not been investigated. In this study, Cyp $19 a 1$ expression significantly decreased during TSP and after TSP when incubation temperatures reduced from $31^{\circ} \mathrm{C}$ to $27^{\circ} \mathrm{C}$ with $1^{\circ} \mathrm{C}$ as an interval (Fig. 2A and B), indicating its temperature dependency. Moreover, we shifted embryos from MPT to FPT or from FPT to MPT, and found that Cyp 19al expression rapidly increased or decreased following the change of incubation temperature (Fig. 2C). These features confirmed that Cyp 19al as a key gene involved in temperature-induced system. The time lag of Cyp19al expression when embryos were shifted from FPT to MPT might be due to Cyp19al expression was upregulated by the continuous activation of STAT3, which is crucial for TSD female pathway (Weber et al., 2020). The STAT3 is long-lived molecules that would be stable for at least 8 hours in cells and may influence the signal transduction process to regulate Cyp19a1 expression (Siewert et al., 1999; Weber et al., 2020). Collectively, these findings confirmed the highly thermo-sensitivity of Cyp $19 \mathrm{al}$ in $\mathrm{M}$. reevesii gonadal cells, indicating its vital role in female pathway connecting temperature and sex of TSD system.

If administered during the TSP, exogenous estrogen and its synthetase aromatase could override the temperature effect to induce the feminization of MPT embryos in TSD reptiles (Janes et al., 2007; Ramsey and Crews, 2009; Matsumoto and Crews, 2012; Warner et al., 2014; Sun et al., 2016), but the process how hormones affect sex-determining genes and further influence individuals' sexual fate remain unclarified. In this study, we treated the embryos with estrogen at stage 16 and found that the Cyp19a1 transcripts expression rapidly upregulated since stage 17 and the gonadal sexual phenotype of MPT embryos at stage 25 permanently feminized. There is no direct experimental evidence show that estrogen would affect the expression of Cyp19al in TSD reptiles. However, it has been proposed that exogenous estrogen might redirect the gonadal trajectory by interacting with the candidate sexdetermining genes like Foxl2, constructing a direct positive feedback regulation of estrogen-Foxl2Cyp19a1-estrogen, which may promote the expression of Cyp19al (Matsumoto and Crews, 2012). Therefore, it is possible that the expression pattern of Cyp 19 al reversed by exogenous estrogen is responsible for the ultimate sex-reversal in $M$. reevesii. At the least, these results suggested that Cyp 19al was required for early ovarian determination in TSD.

In GSD species (e.g., chicken and Chinese soft-shelled turtle), aromatase inhibitor or Cyp19a1 lossof-function treatments induced a permanent female-male sex reversal, which was characterized by the formation of bilateral testis with the spermatogenesis ability and an external male phenotype (Vaillant et al., 2001; Bao et al., 2017; Jin et al., 2020). As aromatase inhibitor (Letrozole) could suppress the function of aromatase by over $98 \%$ (Lamb and Adkins, 1998; Simpson et al., 2004), aromatase inhibitor treatment is a reliable alternative method to identify the function of Cyp 19a1. In this study, suppression of Cyp 19al through aromatase inhibitor treatment in M. reevesii indeed induced a female-to-male sex reversal, which reflected in the high expression of male-specific marker genes and the medulla localization of germ cells (Fig. 3). It's also consistent with the previous studies that aromatase inhibitor administered during the TSP could induce the masculinization of FPT embryo in TSD reptiles (Lance and Bogart, 1992; Wibbels and Crews, 1994; 
213 Ge et al., 2017). These findings demonstrated that, like its role in chicken and Chinese soft-shelled

214 turtle, Cyp19al was necessary for female determination process in TSD turtle M. reevesii.

215 Our study provides unequivocal evidences for the critical function of Cyp19al in the female pathway

216 of turtles, but questions remain. Which factors will be the direct upstream regulator of Cyp19a1? The

217 transcription factor Foxl2, a classical sex-determining gene (De Baere et al., 2001; Schmidt et al.,

218

219

220

221

222

223

224

225

226 2004; Uda et al., 2004; Boulanger et al., 2014), can be one of direct upstream regulators, because the transcription of Cyp19al could be directly promoted by Foxl2 in fish, goat and human (Pannetier et al., 2006; Fleming et al., 2010; Bertho et al., 2018). Another possible upstream regulator is the promoter methylation dynamics of Cyp 19al, which significantly correlated with temperature change and Cyp19al expression (Matsumoto et al., 2013; Parrott et al., 2014; Matsumoto et al., 2016). In addition, $N R F 2$, a redox-sensitive transcription factor, is also reported directly promotes Cyp19al expression during syncytiotrophoblast differentiation (Muralimanoharan et al., 2018). Therefore, which genetic factors directly regulate Cyp19al expression may be the key next step to elucidate the mechanism of female pathway in TSD system.

227 In summary, we have demonstrated that Cyp19al is a key female sex-determining gene in a fresh water turtle $M$. reevesii, which exhibited TSD. This is the first time to identify the function of Cyp19al in a TSD reptile, confirming the importance of Cyp19al in the female sex determination process, thereby shedding new light on the elusive TSD molecular mechanism.

\section{MATERIALS AND METHODS}

\section{$232 \quad 4.1$ Sequence homology comparation and phylogenetic tree construction}

233 The full-length coding sequence of $M$. reevesii Cyp19al have been accessed from previous study 234 (accession number KU821113). Alignment of deducted amino acid sequences were carried out by ClustalX and GENEDOC. Multiple amino acid alignments for the tree construction were performed using ClustalW and the phylogenetic tree was constructed using the neighbor-joining method in Mega 6.0. The accession numbers of amino acid sequences used in the phylogenetic are as follows: Homo sapiens (AMNP_000094.2); Mus musculus (NP_031836.1); Gallus gallus (NP_001001761.2); Alligator mississippiensis (XM_019477372.1); Pelodiscus sinensis (XP_006135137.1); Anolis carolinensis (XP_016852003.1); Python bivittatus (XP_007422249.1); Xenopus tropicalis (NP_001090630.1); Andrias davidianus (ALL29317.1); Danio rerio (NP_571229.3).

\subsection{Tissue-specific expression of Cyp19a1}

Adult fresh water turtles (M. reevesii) were obtained from the Hanshou Turtle Farm (Hunan, China). In order to examine the tissue-specific expression of Cyp19a1, we collected the heart, liver, brain, intestine, skin, kidney, lung, testis and ovary of male and female adult turtles for RT-PCR analysis. And adult testes and ovaries were separately sectioned for Hematoxylin and Eosin staining.

\subsection{Egg collection and incubation}

248 Freshly laid $M$. reevesii turtle eggs were still obtained from the Turtle Farm. For various incubation experiments, fertilized eggs were randomized in plastic boxes with moist vermiculite and placed in incubators at different temperatures with a water potential of $-220 \mathrm{kPa}$. In this species, incubation of eggs at $26^{\circ} \mathrm{C}(\mathrm{MPT})$ generates all males, whereas incubation at $32^{\circ} \mathrm{C}$ (FPT) generates all females 
254

255

256

257

258

259

260

261

262

263

264

265

266

267

268

269

270

271

272

273

274

275

276

277

278

279

280

281

282

283

284

285

286

287

288

289

290

291

292

293

294

295

shifted at developmental stage 16 from an incubator kept at $26^{\circ} \mathrm{C}$ to another kept at $32^{\circ} \mathrm{C}$ and vice versa. For temperature gradient incubation experiments, groups of eggs were placed into incubators held at $25^{\circ} \mathrm{C}, 26^{\circ} \mathrm{C}, 27^{\circ} \mathrm{C}, 28^{\circ} \mathrm{C}, 29^{\circ} \mathrm{C}, 30^{\circ} \mathrm{C}, 31^{\circ} \mathrm{C}$ and $32^{\circ} \mathrm{C}$. Embryos were staged by refer to criteria established by Greenbaum (2002) in Trachemys scripta. We sampled gonads at stages 16, 17, 18, 19, 20, 21 and/or 25 from embryos incubated at MPT, FPT, MPT $\rightarrow$ FPT and FPT $\rightarrow$ MPT and gonads at stages 18 and 21 from embryos incubated at gradient temperature of $25^{\circ} \mathrm{C}-32^{\circ} \mathrm{C}$ for qRT-PCR analysis.

\subsection{Exogenous estrogen and aromatase inhibitor treatments}

In order to examine the role of Cyp19a1, a steroid estrogen ( $\beta$-estradiol, E8875, Sigma) was be administered to eggs incubating at MPT $\left(26^{\circ} \mathrm{C}\right)$, meanwhile a non-steroidal aromatase inhibitor (letrozole, PHR1540, Sigma) was administered to eggs incubating at FPT $\left(32^{\circ} \mathrm{C}\right)$. $\beta$-estradiol or letrozole were dissolved in $95 \%$ ethanol at a concentration of $10 \mu \mathrm{g} / \mu \mathrm{l}$, and $10 \mu \mathrm{l}$ of the drug was applied topically to the eggshell in the region adjacent to the embryo at stage 16. Controls were treated with $10 \mu \mathrm{l}$ of $95 \%$ ethanol. Gonad-mesonephros complexes were dissected from estrogen or aromatase inhibitor treated and control embryos at stage 25 for histology and immunohistochemistry. And gonads treated with estrogen were separated from the adjacent mesonephros at stages 17, 18, 19, 21, 25, and preserved for qRT-PCR analysis.

\subsection{RNA extraction and qRT-PCR}

Gonads from embryos in each group were harvested for RNA extraction using TRIzon Reagent (CW0580, Cwbiotech) according to a routine protocol. First-strand cDNA was synthesize using $0.5 \mu \mathrm{g}$ RNA and EasyQuick RT MasterMix (CW2019, Cwbiotech) based upon the manufacturer's protocol. Quantitative real-time PCR reactions were performed using Roche LightCycler 480 system with a SsoFast EvaGreen Supermix (\#1725201, Bio-Rad). Each sample was run in triplicate. After normalization with $\beta$-actin, relative RNA levels in samples were calculated by the comparative threshold cycle $(\mathrm{Ct})$ method (Schmittgen and Livak, 2008). The sequences of primers for PCR are listed in Table $\mathrm{S} 1$.

\subsection{Hematoxylin and Eosin (H\&E) staining}

Embryo gonad-mesonephros complexes and adult gonads were immersed in $4 \%$ paraformaldehyde (PFA) overnight at $4{ }^{\circ} \mathrm{C}$, and transferred to $70 \%$ ethanol. Tissues were weighing paper wrapped and placed in processing cassettes, dehydrated through a serial alcohol gradient, embedded in paraffin wax blocks and sectioned. Before staining, 5- $\mu \mathrm{m}$-thick tissue sections were dewaxed in xylene, rehydrated through decreasing concentrations of ethanol, and washed in PBS. And then stained with Hematoxylin and Eosin (H\&E). After staining, sections were dehydrated through increasing concentrations of ethanol and xylene, and sealed with Permount TM Mounting Medium.

\subsection{Immunofluorescence}

Gonad-mesonephros complexes from turtle embryos of indicated stages were still immersed in $4 \%$ PFA overnight at $4^{\circ} \mathrm{C}$, then moved through a $\mathrm{MeOH}$ gradient, embedded in paraffin wax and sectioned. Paraffin sections $(\sim 5 \mu \mathrm{m})$ were first deparaffinized, and antigens were unmasked by microwaving sections in $10 \mathrm{mM} / \mathrm{L}$ citrate buffer, $\mathrm{pH} 6.0$ (15 minutes). Sections were covered with primary antibodies and incubated overnight at $4^{\circ} \mathrm{C}$ or 1 hour at room temperature. The primary antibodies used in this analysis included rabbit anti-Sox9 (AB5535, Chemicon, 1:1000), rabbit antiVasa (ab13840, Abcam, 1:200) and mouse anti- $\beta$-catenin (C7207, Sigma, 1:250). Secondary 
antibodies Alexa Fluor 594 donkey anti-rabbit IgG (A21207, Invitrogen) and Alexa Fluor 594 donkey anti-mouse IgG (A21203, Invitrogen), Alexa Fluor 488 donkey anti-rabbit IgG (A21206, Invitrogen), Alexa Fluor 488 donkey anti-mouse IgG (A21202, Invitrogen), which all diluted at

299 1:250, were used to detect primary antibodies. Nuclei were stained with DAPI. Gonad sections were

300 imaged by confocal microscope (A1 Plus, Nikon).

\section{Statistical analyses}

302 Each experiment was independently repeated at least three times. All data are expressed as the mean

$303 \pm$ s.d. Student's unpaired t-test was used to test significance $(* \mathrm{P}<0.05 ; * * \mathrm{P}<0.01 ; * * * \mathrm{P}<0.001 ;$ n.s.,

304 no significance).

\section{Data availability}

306 All data necessary for confirming the conclusions in this paper are included in this article and

307 accompanying figures and tables.

\section{$308 \quad 7 \quad$ Acknowledgements}

309 We thank Hua Ye, Qiong Zhang, Hong-xin Xie, Chun-rong Mi, Zi-han Ding and Ming-shuo Qing for 310 their assistance on turtle eggs collection and incubation. Research was performed under approvals 311 from the Animal Ethics Committee at the Institute of Zoology, Chinese Academy of Sciences 312 (IOZ14001). This work was supported by the National Natural Science Foundation of China (Grant 313 No. 32030013, Grant No. 31821001).

\section{Author contributions}

315 Peng-fei Wu, Xi-feng Wang and Wei-guo Du conceived and designed the study; Peng-fei Wu and 316 Xi-feng Wang performed the experiments; Peng-fei Wu and Xi-feng Wang analyzed data; and Peng317 fei Wu, Xi-feng Wang, Fei Gao and Wei-guo Du cowrote the manuscript. All authors read and 318 approved the manuscript. The authors declare no competing or financial interests.

\section{Reference}

Bao, H., H. Cai, W. Han, H. Zhang, W. Sun et al., 2017 Functional characterization of Cyp19a1 in female sexual differentiation in Pelodiscus sinensis. SCIENTIA SINICA Vitae 47: 640-649.

Bertho, S., A. Herpin, A. Branthonne, E. Jouanno, A. Yano et al., 2018 The unusual rainbow trout sex determination gene hijacked the canonical vertebrate gonadal differentiation pathway. Proceedings of the National Academy of Sciences of the United States of America 115: 12781-12786.

Boulanger, L., M. Pannetier, L. Gall, A. Allais-Bonnet, M. Elzaiat et al., 2014 FOXL2 is a female sex-determining gene in the goat. Current Biology 24: 404-408.

Britt, K. L., A. E. Drummond, M. Dyson, N. G. Wreford, M. E. Jones et al., 2001 The ovarian phenotype of the aromatase knockout (ArKO) mouse. Journal of Steroid Biochemistry \& Molecular Biology 79: 181-185.

Charnier, M., 1966 Action of temperature on sex-ratio in Agama agamas (Agamidae, Lacertilien) embryo Comptes Rendus Des Seances De La Societe De Biologie Et De Ses Filiales 160: 620-622.

Crews, D., and J. M. Bergeron, 1994 Role of reductase and aromatase in sex determination in the red-eared slider (Trachemys-scripta), a turtle with temperature-dependent sex determination. Journal of Endocrinology 143: 279289.

Czerwinski, M., A. Natarajan, L. Barske, L. L. Looger and B. Capel, 2016 A timecourse analysis of systemic and gonadal effects of temperature on sexual development of the red-eared slider turtle Trachemys scripta elegans. Developmental Biology 420: 166-177.

De Baere, E., M. J. Dixon, K. W. Small, E. W. Jabs, B. P. Leroy et al., 2001 Spectrum of FOXL2 gene mutations in blepharophimosis-ptosis-epicanthus inversus (BPES) families demonstrates a genotype-phenotype correlation. 
Human Molecular Genetics 10: 1591-1600. granulosa cells from normal and polycystic ovaries. The Journal of Clinical Endocrinology and Metabolism 49: 514-519.

Ferguson, M. W. J., and T. Joanen, 1982 Temperature of egg incubation determines sex in Alligator mississippiensis. Nature 296: 850-853.

Fernandino, J. I., R. S. Hattori, T. Shinoda, H. Kimura, P. H. Strobl-Mazzulla et al., 2008 Dimorphic expression of Dmrt1 and Cyp19a1 (ovarian Aromatase) during early gonadal development in Pejerrey, Odontesthes bonariensis. Sexual Development 2: 316-324.

Fleming, N. I., K. C. Knower, K. A. Lazarus, P. J. Fuller, E. R. Simpson et al., 2010 Aromatase is a direct target of FOXL2: $\mathrm{C} 134 \mathrm{~W}$ in granulosa cell tumors via a single highly conserved binding site in the ovarian specific promoter. Plos One 5: e14389.

Gabriel, W. N., B. Blumberg, S. Sutton, A. R. Place and V. A. Lance, 2001 Alligator aromatase cDNA sequence and its expression in embryos at male and female incubation temperatures. Journal of Experimental Zoology 290: 439448.

Ge, C., J. Ye, C. Weber, W. Sun, H. Zhang et al., 2018 The histone demethylase KDM6B regulates temperature-dependent sex determination in a turtle species. Science 360: 645-648.

Ge, C., J. Ye, H. Zhang, Y. Zhang, W. Sun et al., 2017 Dmrt1 induces the male pathway in a turtle species with temperaturedependent sex determination. Development 144: 2222-2233.

Greenbaum, E., 2002 A standardized series of embryonic stages for the emydid turtle Trachemys scripta. Canadian Journal of Zoology-Revue Canadienne De Zoologie 80: 1350-1370.

Gutzke, W. H. N., and J. J. Bull, 1986 Steroid-hormones reverse sex in turtles. General and Comparative Endocrinology 64: 368-372.

Hamilton, K. J., Y. Arao and K. S. Korach, 2014 Estrogen hormone physiology: Reproductive findings from estrogen receptor mutant mice. Reproductive Biology 14: 3-8.

Hou, L., 1985 Sex determination by temperature for incubation in Chinemys reevesii (In Chinese with English abstract). Acta Herpetol Sin 4: 130.

Janes, D. E., D. Bermudez, L. J. Guillette and M. L. J. J. o. H. Wayne, 2007 Estrogens induced male production at a femaleproducing temperature in a reptile (Leopard Gecko, Eublepharis macularius) with temperature-dependent sex determination. Journal of Herpetology 41: 9-15.

Jin, K., Q. Zuo, J. Song, Y. Zhang, G. Chen et al., 2020 CYP19A1 (aromatase) dominates female gonadal differentiation in chicken (Gallus gallus) embryos sexual differentiation. Bioscience Reports 40: BSR20201576.

Kitano, T., K. Takamune, Y. Nagaham and S. Abe, 2000 Aromatase inhibitor and 17 alpha-methyltestosterone cause sexreversal from genetical females to phenotypic males and suppression of P450 aromatase gene expression in Japanese flounder (Paralichthys olivaceus). Molecular Reproduction and Development 56: 1-5.

Lamb, H. M., and J. C. Adkins, 1998 Letrozole. Drugs 56: 1125-1140.

Lambeth, L. S., D. Cummins, T. J. Doran, A. H. Sinclair and C. A. Smith, 2013 Overexpression of Aromatase alone is sufficient for ovarian development in genetically male chicken embryos. Plos One 8: e68362.

Lance, V. A., and M. H. Bogart, 1992 Disruption of ovarian development in alligator embryos treated with an aromatase inhibitor. General and Comparative Endocrinology 86: 59-71.

Matsumoto, Y., A. Buemio, R. Chu, M. Vafaee and D. Crews, 2013 Epigenetic Control of Gonadal Aromatase (cyp19a1) in Temperature-Dependent Sex Determination of Red-Eared Slider Turtles. PLOS ONE 8: e63599.

Matsumoto, Y., and D. Crews, 2012 Molecular mechanisms of temperature-dependent sex determination in the context of ecological developmental biology. Molecular and Cellular Endocrinology 354: 103-110.

Matsumoto, Y., B. Hannigan and D. Crews, 2016 Temperature shift alters DNA methylation and histone modification patterns in gonadal Aromatase (Cyp19a1) gene in species with temperature-dependent sex determination. Plos One 11: e0167362.

Mawaribuchi, S., N. Ikeda, K. Fujitani, Y. Ito, Y. Onuma et al., 2014 Cell-mass structures expressing the Aromatase gene Cyp19a1 lead to ovarian cavities in Xenopus laevis. Endocrinology 155: 3996-4005.

Meseke, M., F. Pröls, C. Schmahl, K. Seebo, C. Kruse et al., 2018 Reelin and aromatase cooperate in ovarian follicle development. 8: 8722.

Muralimanoharan, S., Y.-T. Kwak and C. R. Mendelson, 2018 Redox-sensitive transcription factor NRF2 enhances trophoblast differentiation via induction of miR-1246 and aromatase. Endocrinology 159: 2022-2033.

Murdock, C., T. J. G. Wibbels and c. endocrinology, 2003 Cloning and expression of aromatase in a turtle with temperaturedependent sex determination. General and comparative endocrinology 130: 109-119.

Nakamoto, M., Y. Shibata, K. Ohno, T. Usami, Y. Kamei et al., 2018 Ovarian aromatase loss-of-function mutant medaka undergo ovary degeneration and partial female-to-male sex reversal after puberty. Molecular and Cellular 
Endocrinology 460: 104-122.

Nelson, E. R., and H. R. Habibi, 2013 Estrogen receptor function and regulation in fish and other vertebrates. General comparative endocrinology 192: 15-24.

Pannetier, M., S. Fabre, F. Batista, A. Kocer, L. Renault et al., 2006 FOXL2 activates P450 aromatase gene transcription: towards a better characterization of the early steps of mammalian ovarian development. Journal of Molecular Endocrinology 36: 399-413.

Parrott, B. B., S. Kohno, J. A. Cloy-Mccoy and L. J. Guillette, Jr., 2014 Gonadal DNA methylation patterning is affected by incubation temperature in the American alligator, a species undergoing temperature-dependent sex determination. Integrative and Comparative Biology 54: E327.

Pieau, C., M. Dorizzi and N. Richard-Mercier, 1999 Temperature-dependent sex determination and gonadal differentiation in reptiles. Cellular and Molecular Life Sciences 55: 887-900.

Radhakrishnan, S., R. Literman, J. Neuwald, A. Severin and N. Valenzuela, 2017 Transcriptomic responses to environmental temperature by turtles with temperature-dependent and genotypic sex determination assessed by RNAseq inform the genetic architecture of embryonic gonadal development. Plos One 12: e0172044.

Ramsey, M., and D. Crews, 2009 Steroid signaling and temperature-dependent sex determination - Reviewing the evidence for early action of estrogen during ovarian determination in turtles. Seminars in Cell \& Developmental Biology 20: 283-292.

Ramsey, M., C. Shoemaker and D. Crews, 2007 Gonadal expression of Sf1 and aromatase during sex determination in the red-eared slider turtle (Trachemys scripta), a reptile with temperature-dependent sex determination. Differentiation 75: 978-991.

Sakata, N., Y. Tamori and M. Wakahara, 2005 P450 aromatase expression in the temperature-sensitive sexual differentiation of salamander (Hynobius retardatus) gonads. International Journal of Developmental Biology 49: 417-425.

Schmidt, D., C. E. Ovitt, K. Anlag, S. Fehsenfeld, L. Gredsted et al., 2004 The murine winged-helix transcription factor Fox12 is required for granulosa cell differentiation and ovary maintenance. Development 131: 933-942.

Schmittgen, T. D., and K. J. Livak, 2008 Analyzing real-time PCR data by the comparative C-T method. Nature Protocols 3: 1101-1108.

Shoemaker-Daly, C. M., K. Jackson, R. Yatsu, Y. Matsumoto and D. Crews, 2010 Genetic network underlying temperaturedependent sex determination is endogenously regulated by temperature in isolated cultured Trachemys scripta gonads. Developmental Dynamics 239: 1061-1075.

Shoemaker, C., M. Ramsey, J. Queen and D. Crews, 2007a Expression of Sox9, Mis, and Dmrt1 in the gonad of a species with temperature-dependent sex determination. Developmental Dynamics 236: 1055-1063.

Shoemaker, C., M. Ramsey, J. Queen and D. Crews, 2007b Expression of Sox9, Mis, and Dmrt1 in the gonad of a species with temperature-dependent sex determination. Dev Dyn 236: 1055-1063.

Siewert, E., W. Muller-Esterl, R. Starr, P. C. Heinrich and F. Schaper, 1999 Different protein turnover of interleukin-6-type cytokine signalling components. European Journal of Biochemistry 265: 251-257.

Sifuentes-Romero, I., H. Merchant-Larios, S. L. Milton, N. Moreno-Mendoza, V. Diaz-Hernandez et al., 2013 RNAimediated gene silencing in a gonad organ culture to study sex determination mechanisms in sea turtle. Genes 4: 293-305.

Simpson, D., M. P. Curran and C. M. Perry, 2004 Letrozole. Drugs 64: 1213-1230.

Simpson, E. R., M. S. Mahendroo, G. D. Means, M. W. Kilgore, M. M. Hinshelwood et al., 1994 Aromatase cytochromep450, the enzyme responsible for estrogen biosynthesis. Endocrine Reviews 15: 342-355.

Spotila, L. D., and S. E. Hall, 1998 Expression of a new RNA-splice isoform of WT1 in developing kidney-gonadal complexes of the turtle, Trachemys scripta. Comparative Biochemistry and Physiology B-Biochemistry \& Molecular Biology 119: 761-767.

Sun, B.-J., T. Li, Y. Mu, J. K. McGlashan, A. Georges et al., 2016 Thyroid hormone modulates offspring sex ratio in a turtle with temperature-dependent sex determination. Proceedings of the Royal Society B: Biological Sciences 283: 20161206.

Tang, W.-Q., Y. Mu, N. Valenzuela and W.-G. Du, 2017 Effects of incubation temperature on the expression of sex-related genes in the Chinese pond turtle, Mauremys reevesii. Sexual Development 11: 307-319.

Tokunaga, E., Y. Hisamatsu, K. Tanaka, N. Yamashita, H. Saeki et al., 2014 Molecular mechanisms regulating the hormone sensitivity of breast cancer. Cancer science 105: 1377-1383.

Uda, M., C. Ottolenghi, M. Deiana, W. Kimber, A. Forabosco et al., 2004 Foxl2 disruption causes mouse ovarian failure by pervasive blockage of follicle development. Human Molecular Genetics 13: 1171-1181.

Vaillant, S., M. Dorizzi, C. Pieau and N. Richard-Mercier, 2001 Sex reversal and aromatase in chicken. Journal of Experimental Zoology 290: 727-740.

Valenzuela, N., and V. Lance, 2004 Temperature-dependent sex determination in vertebrates. Smithsonian Books, Washington, DC.

Valenzuela, N., J. L. Neuwald and R. Literman, 2013 Transcriptional evolution underlying vertebrate sexual development. 
Developmental Dynamics 242: 307-319.

Valenzuela, N., and T. Shikano, 2007 Embryological ontogeny of Aromatase gene expression in Chrysemys picta and Apalone mutica turtles: comparative patterns within and across temperature-dependent and genotypic sexdetermining mechanisms. Development genes evolution 217: 55-62.

Warner, D. A., E. Addis, W.-g. Du, T. Wibbels and F. J. Janzen, 2014 Exogenous application of estradiol to eggs unexpectedly induces male development in two turtle species with temperature-dependent sex determination. General and comparative endocrinology 206: 16-23.

Weber, C., Y. Zhou, J. G. Lee, L. L. Looger, G. Qian et al., 2020 Temperature-dependent sex determination is mediated by pSTAT3 repression of Kdm6b. 368: 303-306.

Western, P. S., J. L. Harry, J. A. M. Graves and A. H. Sinclair, 2000 Temperature-dependent sex determination in the American alligator: expression of SF1, WT1 and DAX1 during gonadogenesis. Gene 241: 223-232.

Wibbels, T., and D. Crews, 1992 Specificity of steroid hormone-induced sex determination in a turtle. Journal of Endocrinology 133: 121-129.

Wibbels, T., and D. Crews, 1994 Putative aromatase inhibitor induces male sex determination in a female unisexual lizard and in a turtle with temperature-dependent sex determination. Journal of Endocrinology 141:295-299.

Yatsu, R., S. Miyagawa, S. Kohno, B. B. Parrott, K. Yamaguchi et al., 2016 RNA-seq analysis of the gonadal transcriptome during Alligator mississippiensis temperature-dependent sex determination and differentiation. BMC Genomics 17: 77 .

\section{Figure legends}

Fig. 1. The sexually dimorphic expression pattern of Cyp19a1 in $M$. reevesii. The mRNA expression of Cyp19al in gonads of different stages (16-25) at MPT $\left(26^{\circ} \mathrm{C}\right)$ and $\mathrm{FPT}\left(32^{\circ} \mathrm{C}\right)$ determined by qRT-PCR analysis; $\beta$-actin was used as a reference gene. Cyp19al exhibited a highly FPT-specific expression pattern in early embryonic gonads. Data are mean \pm s.d.; $n \geqslant 3.1$

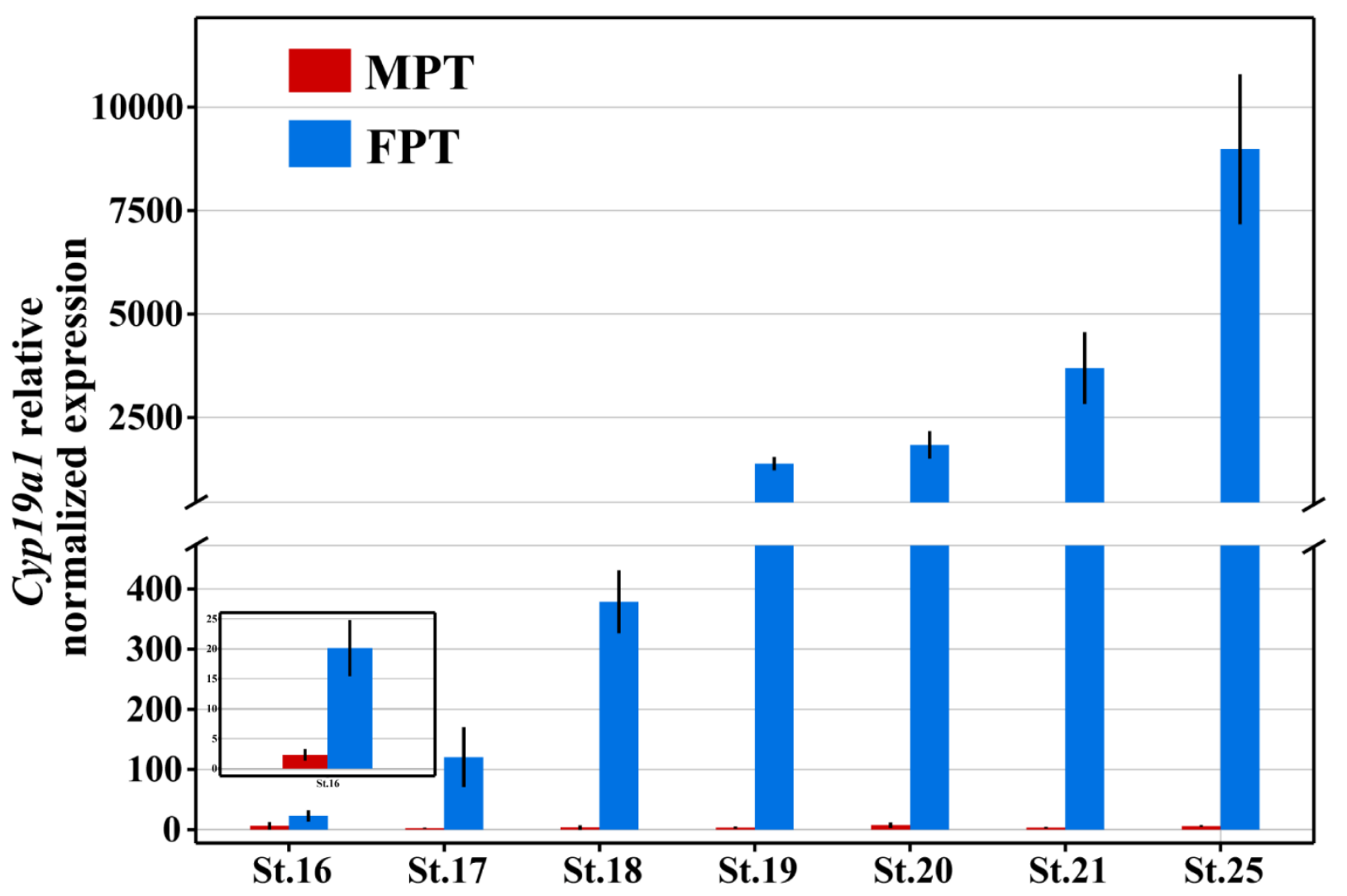


479

480

481

482

483

484

485

486 487

Fig. 2. Thermo-sensitivity of Cyp19a1 in M. reevesii. (A, B) qRT-PCR analysis of Cyp19a1 in gonads at stage 18 or stage 21 of gradient temperatures. The mRNA expression of Cyp 19al in embryonic gonads exhibited temperature dependence, no matter during the TSP (A) or after (B). (C) Time-course response of Cyp19al expression to temperature shifts from either MPT $\rightarrow$ FPT or FPT $\rightarrow$ MPT. Embryos were shifted at stage 16, and gonads were dissected for qRT-PCR analysis at stages 16-21. Cyp19a1 expression in gonads with temperature shifts from MPT $\rightarrow$ FPT responded rapidly to the new temperatures, with rapid expression changes occurring at stage 17. In the opposite shift (FPT $\rightarrow$ MPT), Cyp 19a1 expression continuously increased a little until stage 19, and then decreased towards the MPT-typical level. Data are mean \pm s.d.; $\mathrm{n} \geqslant 3$. $\mathrm{P}<0.05$.

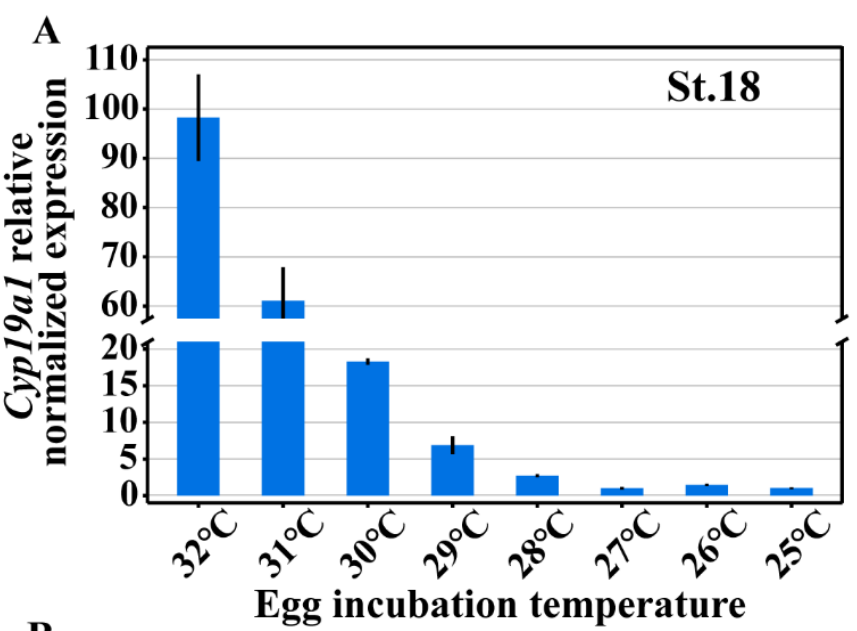

B

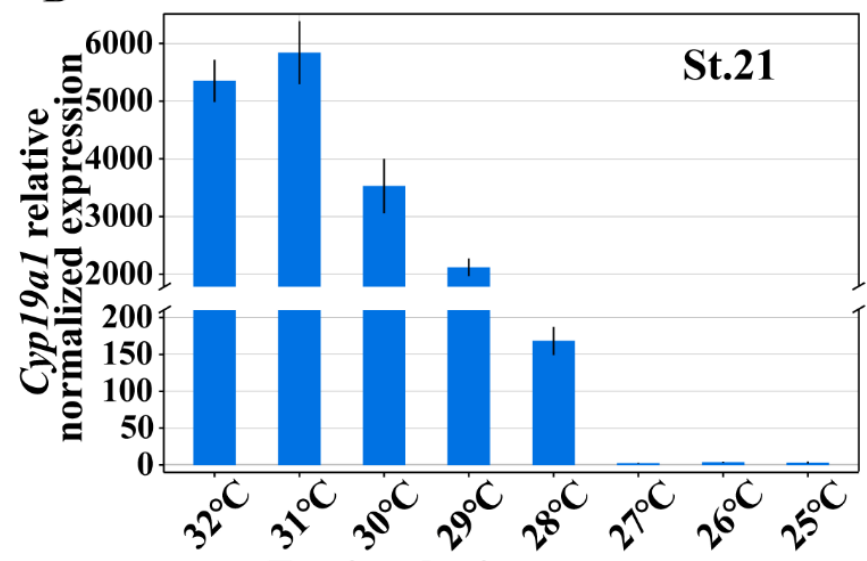

Egg incubation temperature

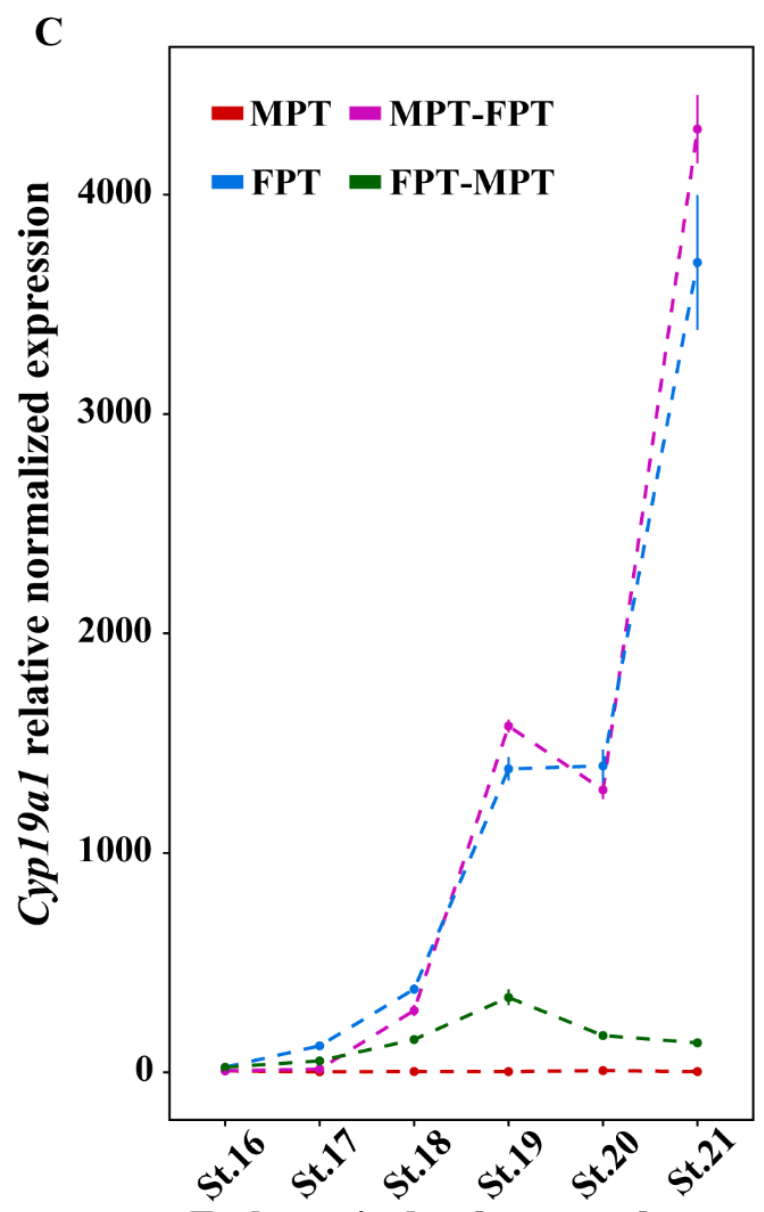

Embryonic developmental stage 
490 Fig. 3. The upregulation of Cyp19a1 in feminized MPT gonads during male-to-female sex

491 reversal. (A-C) Gonads (outlined by white dotted lines) on top of mesonephros of MPT, feminized

492 MPT and FPT embryos at stage 25. Gd, gonad; Ovi, oviduct. Scale bars: $1 \mathrm{~mm}$. (D-F) H\&E staining

493 of gonadal sections from MPT, feminized MPT and FPT embryos at stage 25 showed that the

494 feminized MPT embryonic gonads had a degenerated medulla and a thickened outer cortex. The

495 dashed line indicates the border between medulla and cortex. pgc, primordial germ cells; sc,

496 seminiferous cord; Cor, cortex; Med, medulla; Scale bars: $50 \mu \mathrm{m}$. (G) qRT-PCR analysis of Foxl2,

497 Rspol, Dmrt1 and Sox9 in gonads from MPT, MPT with estrogen treatment and FPT embryos at

498 stage 25. Significant upregulation of Foxl2 and Rspol expression and strong downregulation of

499 Dmrt1 and Sox9 expression were observed in stage 25 MPT gonads with estrogen treatment. (H-J'')

500 Protein localization of Sox9 in MPT, feminized MPT and FPT gonadal sections at stage 25. Sox9

501 expression was totally lost in feminized MPT gonads. Scale bars: $50 \mu \mathrm{m}$. (K-M ${ }^{\prime}$ ) A female-typical

502 distribution of germ cells was observed in MPT gonads with estrogen treatment at stage 25,

503 determined by Vasa and $\beta$-catenin immunostaining. Scale bars: $50 \mu \mathrm{m}$. (N) qRT-PCR analysis of

504 Cyp19al in gonads from MPT, feminized MPT and FPT embryos at stages 17, 18, 19, 21 and 25.

505 Cyp19al exhibited a rapid response to estrogen treatment and was rapidly upregulated at stage 17.

506 Data are mean \pm s.d.; $* \mathrm{P}<0.05 ; * * \mathrm{P}<0.01 ; * * * \mathrm{P}<0.001 ; \mathrm{n} \geqslant 3$. 

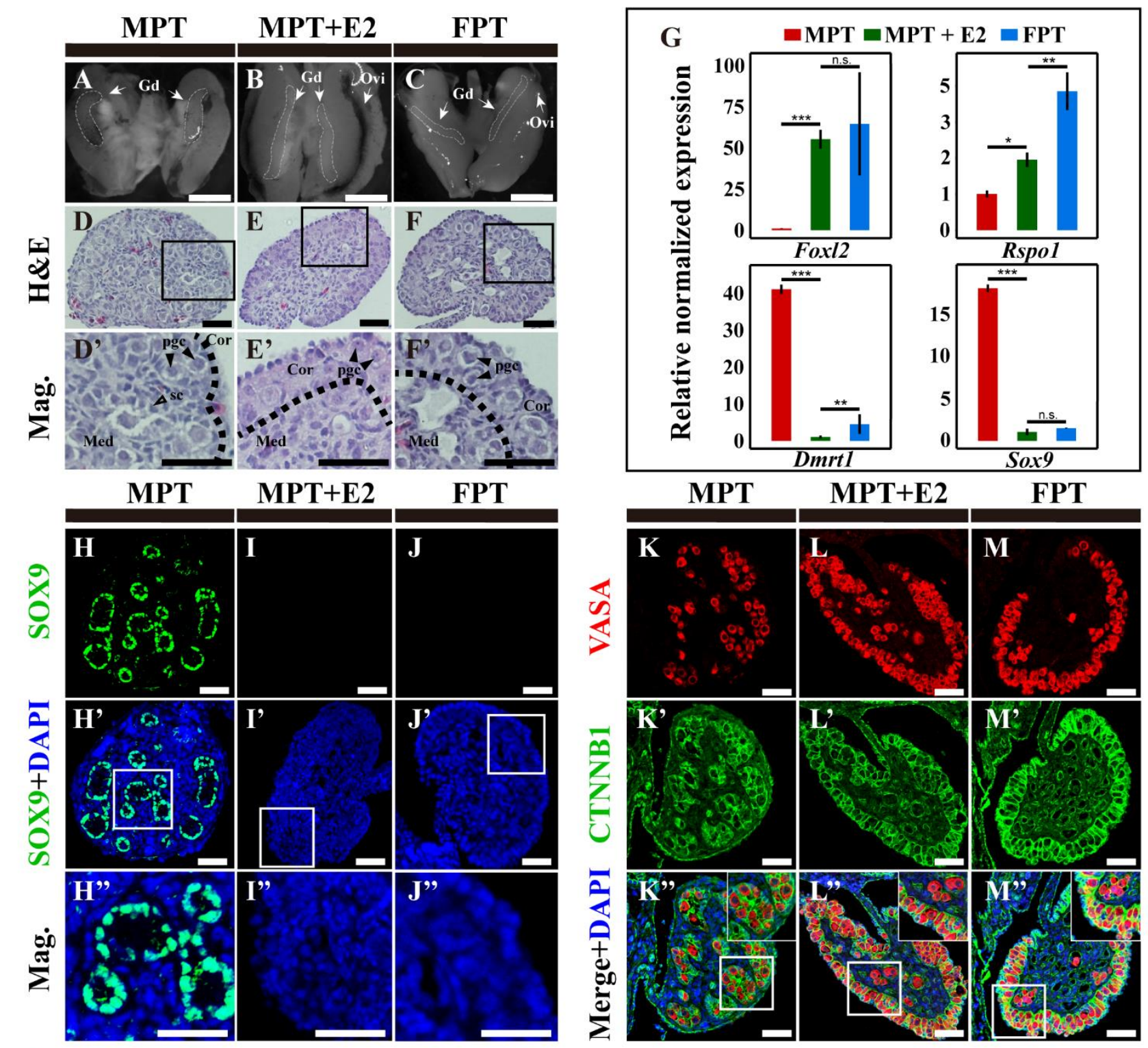

Na

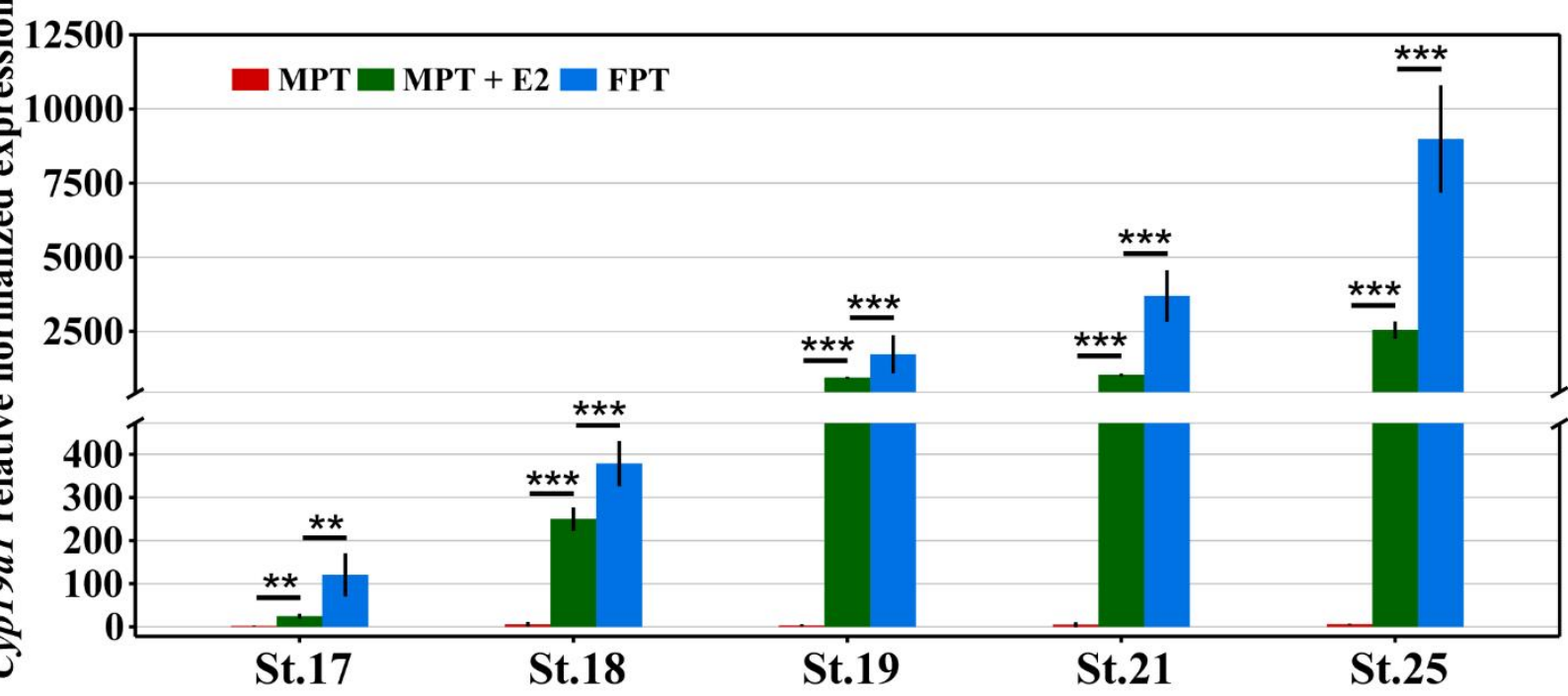


508 Fig. 4. Masculinization of FPT embryos following aromatase inhibitor treatment. (A-C)

509 Representative images of the gonad-mesonephros complexes from MPT, FPT with aromatase

510 inhibitor treatment and FPT embryos at stage 25. Gd, gonad; Ovi, oviduct. Scale bar: $1 \mathrm{~mm}$. (D-F)

511 H\&E of gonadal sections from MPT, FPT with aromatase inhibitor treatment and FPT embryos at

512 stage 25. The FPT gonads with aromatase inhibitor treatment displayed a testis-like phenotype,

513 characterized by a thickened outer cortex and degenerated or absent testis cords. The dashed black

514 line indicates the border between medulla and cortex. Scale bars: $50 \mu \mathrm{m}$. (G-I') Immunofluorescence

515 of Sox 9 and $\beta$-catenin in gonadal sections of MPT, FPT with aromatase inhibitor treatment and FPT

516 embryos at stage 25. Sox9 protein expression was robustly expressed in FPT gonads following

517 aromatase inhibitor treatment. (J-L' ) Immunofluorescence of Vasa in MPT, FPT with aromatase

518 inhibitor treatment and FPT gonads at stage 25. Inhibition of aromatase in FPT gonads led to male-

519 like distribution of germ cells within seminiferous cords. Scale bars: $50 \mu \mathrm{m}$. 


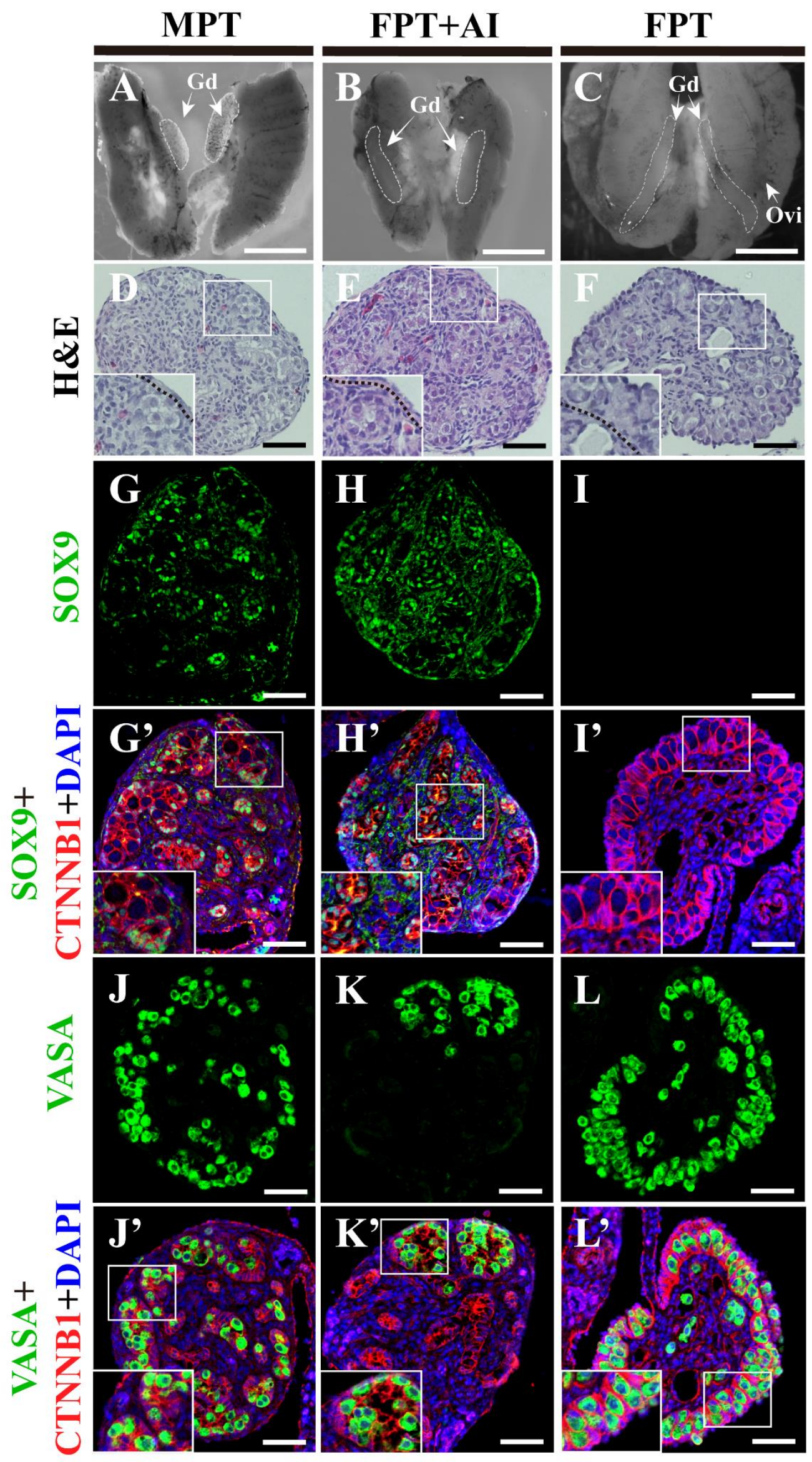




\section{Supplementary Material}

522 Figure S1. Sequence and phylogenetic analyses of $M$. reevesii Cyp19a1. (A) the complete cDNA

523 sequence of $M$. reevesii Cyp19al and deduced amino acid sequence. The start codon ATG was

524 underlined, and the stop codon was indicated by an asterisk. The highly conserved cytochrome P450

525 domain was in shadow. (B) Alignment of amino acid sequence of M. reevesii Cyp19al with those

526 from other typical species. (C) Cyp19al phylogenetic tree analysis of M. reevesii and other typical

527 species based on Neighbor-Joining (N-J) method. Numbers at major branche nodes were bootstraps

528 percentage values based on 1000 replicates. Each branch length scale in terms of genetic distance

529 was indicated at the bottom of tree. 
bioRxiv preprint doi: https://doi.org/10.1101/2021.05.17.444498; this version posted May 17, 2021. The copyright holder for this preprint (which was not certified by peer review) is the author/funder, who has granted bioRxiv a license to display the preprint in perpetuity. It is made available under aCC-BY-NC-ND 4.0 International license.

A

gggetcagttacaggacctgcaaaggggttgcaaggtaccgattaaaggtgtcttagcattttgaaactttctgectctttccatattttcccccctaaaggcaaagtagactctctta 120 gaaaagatgctactggaaaccetgaatctgatgcattacaatatcaccagtgtggtgccagaagtgatgccaactgccaccgtgeccatactcctcctcgtgtgctttcttttcctaatc 240

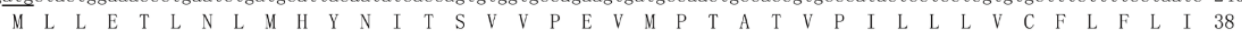
tggaattatgaagaaacatcatcaataccagggcctggtttctgtatgggaattggtcccetcatttcacatgggagattcctttggatgggagtgggcaatgcctgcaactattacaat 360

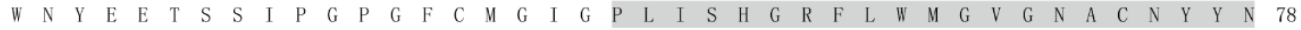
aagatgtatggagaatttatgagagtctggatcagtggagaggaaacactcattattagcagatcctccagtatcttccatgtaatgaaacatgggcaatatagctgtagatttggaagc 480

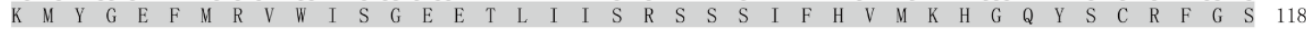
aaacttgggttacagtgcattggcatgcatgaaaatggtattatatttaataataacccagcactctggaaagaattcgaccttttttcaccaaagctttgtccggtcctggtctcgtg 600

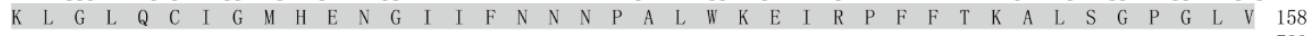
cgtatgatagcaatttgtgttgaatcaacgaaagaccatctggacagattggagaatgtgactgctggactgggaaacatcaatgtgctgaattttatgagacagatcacactggacact 720

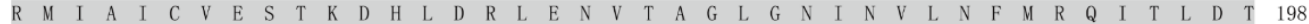
tctaacacactctttctagggatccetttggatgaaaatgccattgtgcttaaaattcagaactactttgatgcttggcaagcacttttgctgaaacctgacatcttctttaaaatttct 840 \begin{tabular}{llllllllllllllllllllllllllllllllllllllllll}
\hline & $N$ & $N$ & T & $L$ & $F$ & $L$ & $G$ & $I$ & $P$ & $L$ & $D$ & $E$ & $N$ & $A$ & $I$ & $V$ & $L$ & $K$ & $I$ & $Q$ & $N$ & $Y$ & $F$ & $D$ & $A$ & $W$ & $Q$ & $A$ & $L$ & $L$ & $L$ & $K$ & $P$ & $D$ & $I$ & $F$ & $F$ & $K$ & $I$ & $S$ & 238 \\
\end{tabular} tggctgtacaagaaatatgaaaaatcagtcaaggatttgaaagaagcaattgaaatcttaatagagcagaaacgacagagactttccactgttgaaaaattggaagagcacatggacttt 960 W gcatcacaactgatttttgcgcagagccgtggagatctgaccggtgagaatgtgaaccagtgtgtgttggagatgatgatagctgcacctgacacgctctctgtgactctcttcttcatg 1080

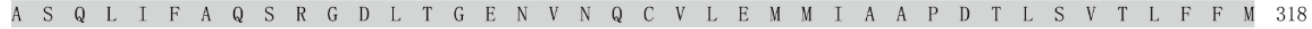
ctagtactgattgcagagcatcctaaagtggaagaagatatgatgaaggaaatccaagctgttataggtgacagagacgtacagagcaatgacatgtcgaacttaaaggttgtggagaat 1200

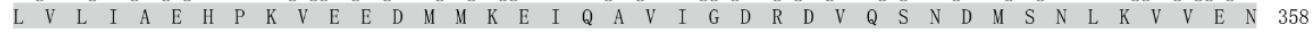
tttat taatgagagtatgagataccagccagttgtggatttggtcatgcgcaaggetttacaggatgatgtaattgatggctatcctgtgaaaagggggacaaacatcattctgaacatt 1320

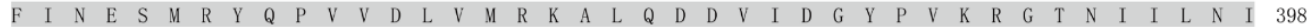
ggacgtatgcataagcttgaattcttcccaaagcctaatgagttttctctcgaaaattttgagaagaatgttcettctcgctactttcagccatttggatttggccetcgtggctgtgtt 1440 $\begin{array}{llllllllllllllllllllllllllllllllllllllllll}G & R & M & H & K & L & E & F & F & P & K & P & N & E & F & S & L & E & N & F & E & K & N & V & P & S & R & Y & F & Q & P & F & G & F & G & P & R & G & C & V & 438\end{array}$ ggaaagt tattgccatggtaatgatgaaggcaatcctggtgactcttctgagacggtatagagtacagacactgaaaggaagaggccttaagaacatccagaaaagcaatgacttatcc 1560 $\begin{array}{llllllllllllllllllllllllllllllllllllllllll}G & K & F & I & A & M & V & M & M & K & A & I & L & V & T & L & L & R & R & Y & R & V & Q & T & L & K & G & R & G & L & K & N & I & Q & K & S & N & D & L & S & 478\end{array}$ atgcacccgaatgaaagacagcccttattggagatgtttttcatgccaagaagaaacatagacaagtgtcaggatgactaacaacagatcagtagtggtctcagagatctaaggtttctg 1680 $\begin{array}{lllllllllllllllllllllllllllllll}\text { M } & H & P & N & E & R & Q & P & L & L & E & M & F & F & M & P & R & R & N & I & D & K & C & Q & D & D & * & & 504\end{array}$ ctgttctagtcacattctgagaaatgtccacccetcctaggtgetgtcacacatactctgaactacacattttgttgetcttaatgeattgetgetctaagtgatcacttattctgetgc 1800 aagcaagaccaggttctggtcatgattcaaactgataaataagtttcaagtatatagacaagtgctgtcttcagttgatgagatgttgcatgcagaaactgtcattactttttttaaa 1920 gcatccaattcaaacagaattctgttctgettctctcactgtgcaagtcctgagtgctctatgcctgcagacatg

B

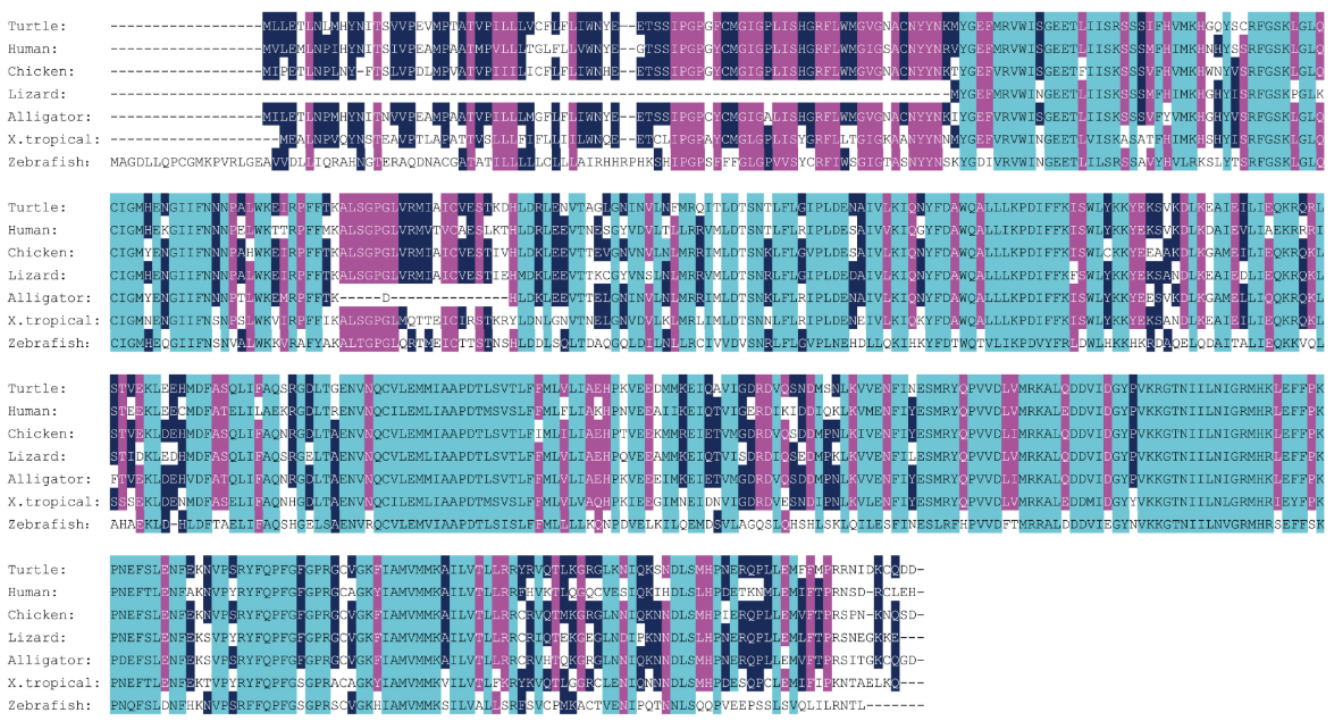

C

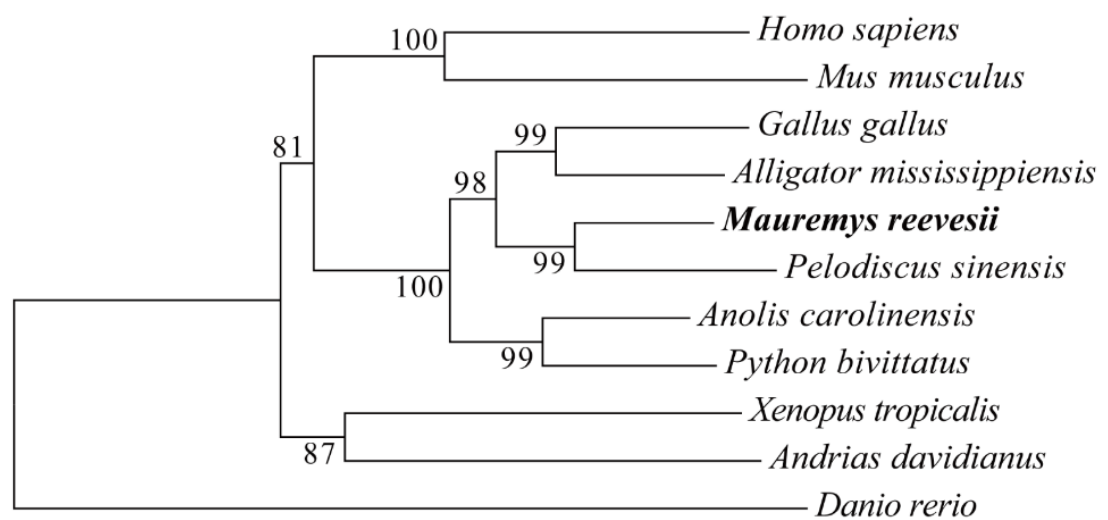

0.05 
531 Figure S2. Cyp19a1 expression and histology of testis and ovary in adult $M$. reevesii. (A) The expression of Cyp19al mRNA in different tissues was analyzed by RT-PCR, which specifically high expressed in ovary instead of testis. (B) H\&E staining of adult testis and ovary sections. sc,

534 seminiferous cord; fol, follicle; scale bars are $50 \mu \mathrm{m}$.

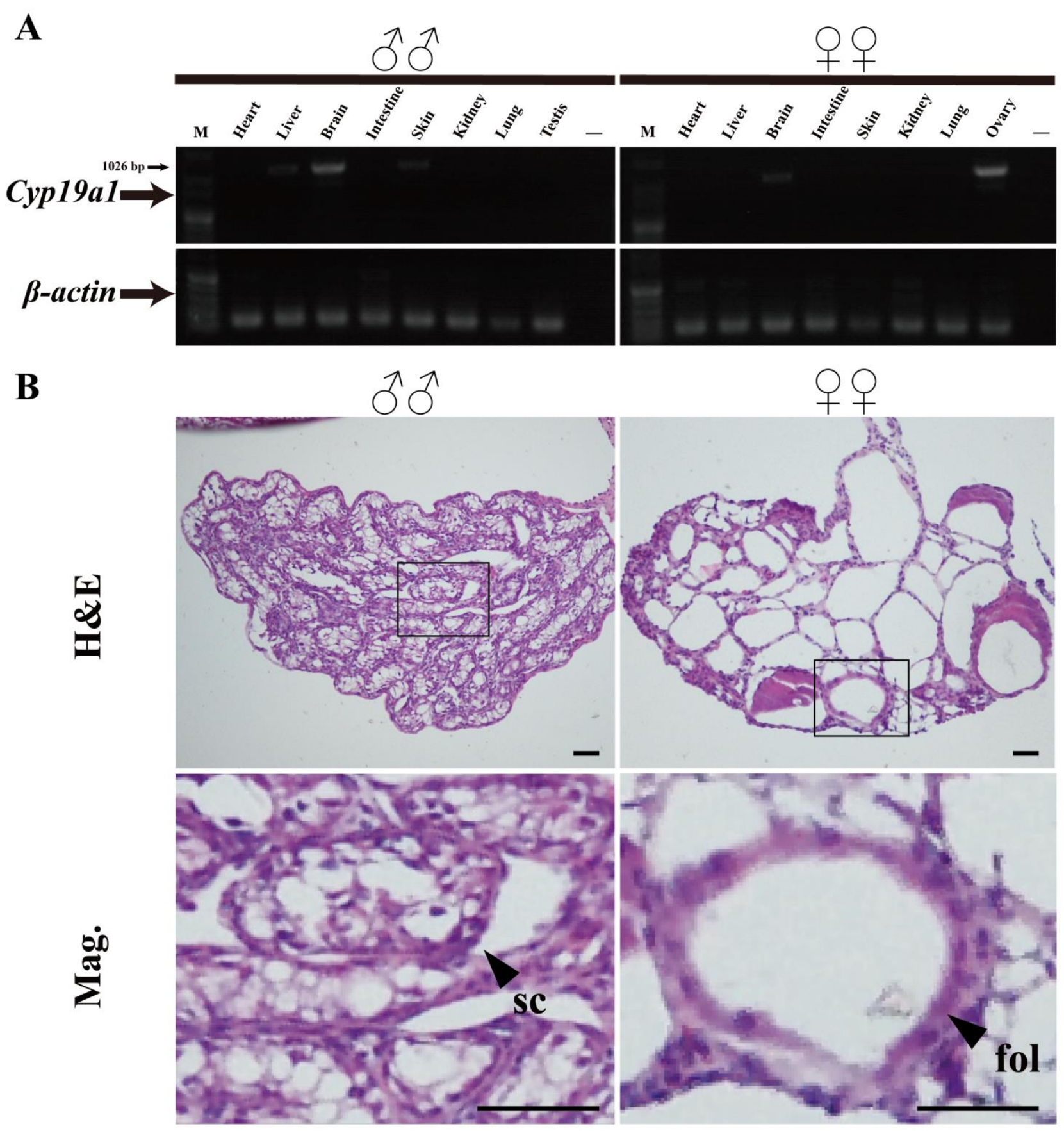


537 Figure S3. Expression of sex-specific marker genes in MPT gonads following estrogen

538 treatment at stage 21. (A-C') Sox9 protein expression in gonadal sections from MPT, MPT with

539 estrogen treatment and FPT embryos. (D-F') The distribution of germ cells in MPT gonads

540 following estrogen treatment, determined by Vasa immunostaining. Scale bars are $50 \mu \mathrm{m}$.
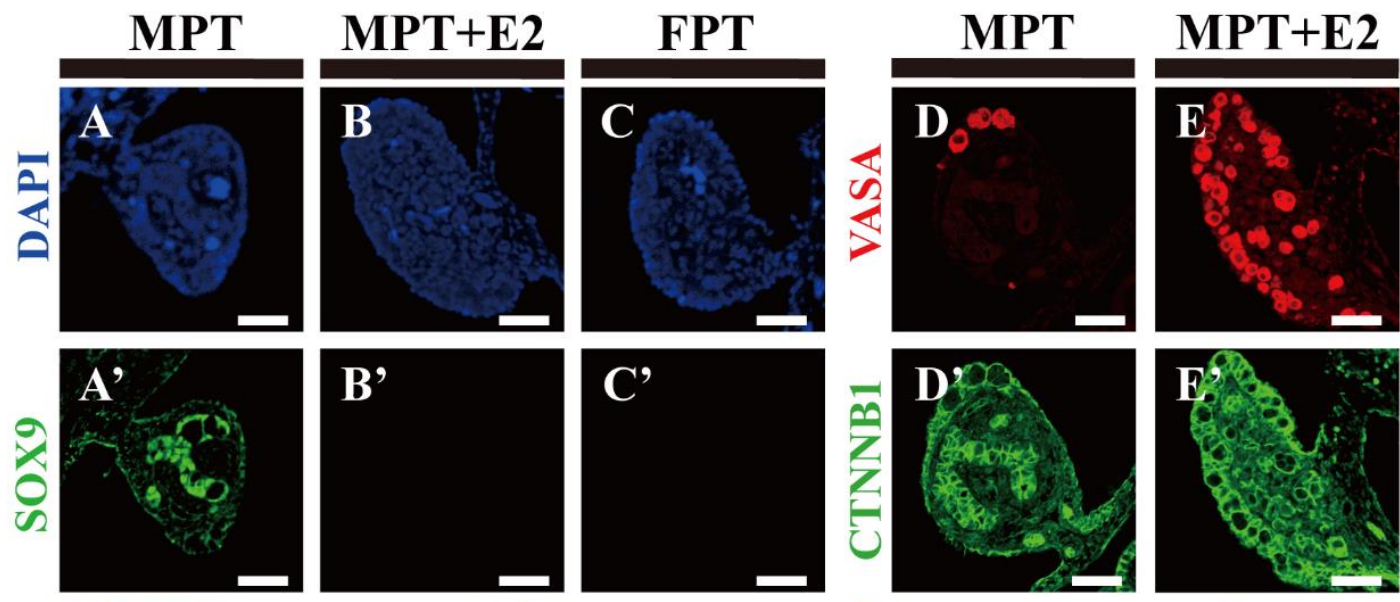

FPT
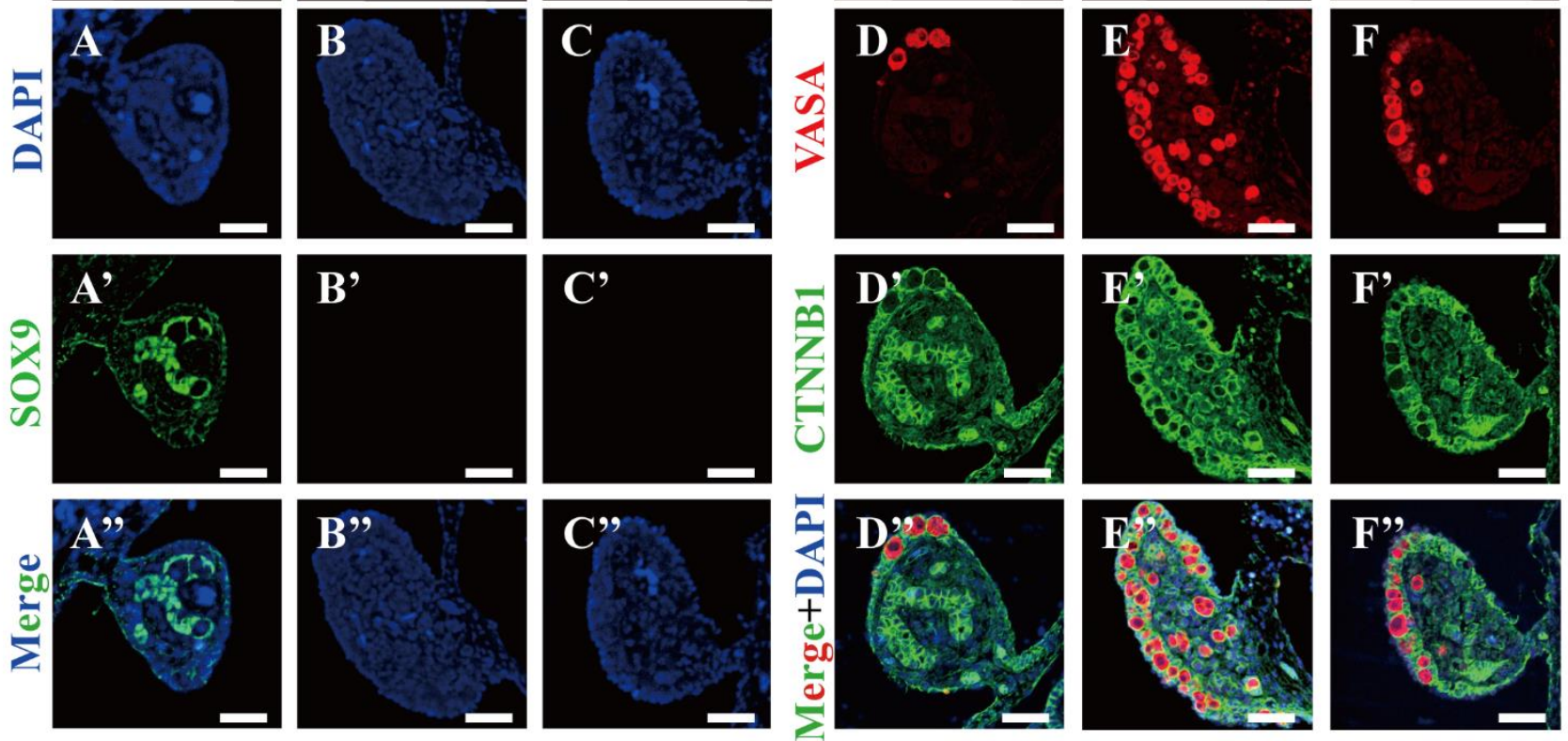
543 Figure S4. Masculinization of FPT M. reevesii embryos following aromatase inhibitor

544 treatment at stage 21. (A-C' ') Immunofluorescence of Sox9 in transverse sections of MPT, FPT

545 with aromatase inhibition and FPT gonads. (D-F') Immunofluorescence of vasa and $\beta$-catenin of

546 MPT, FPT with aromatase inhibition and FPT gonads. Scale bars: $50 \mu \mathrm{m}$.
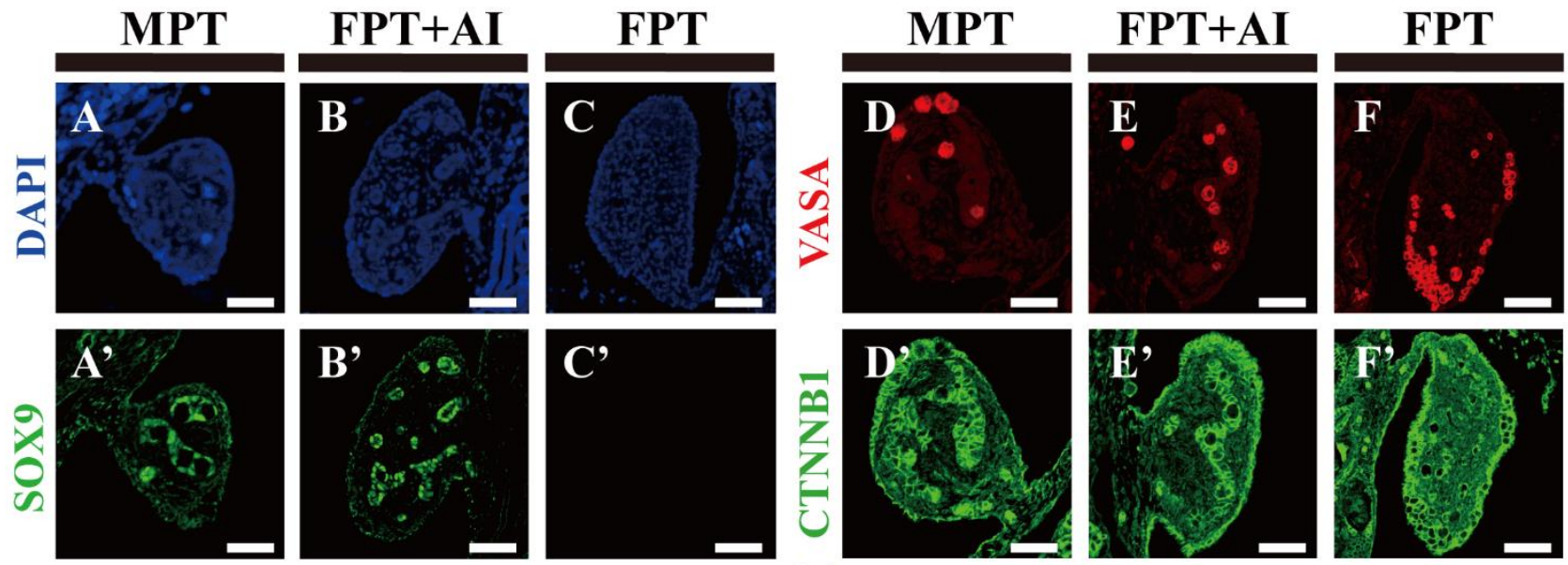

547
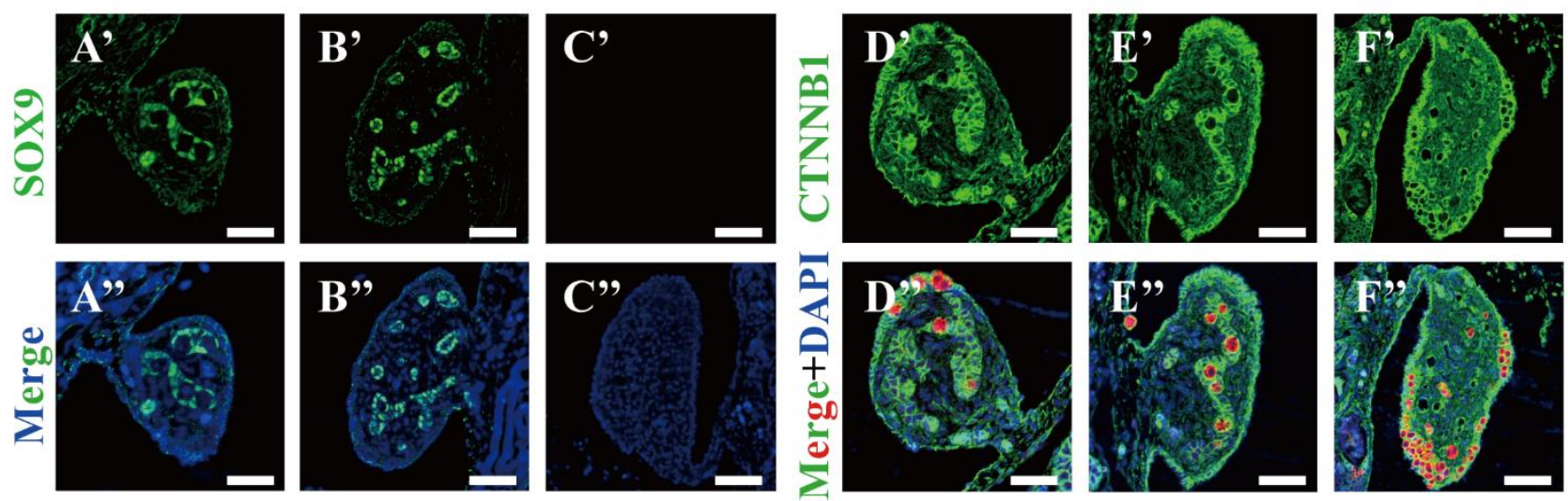

548 
549 Table S1. Primer list.

$\begin{array}{ll}\text { Experiments } & \begin{array}{l}\text { Direction of } \\ \text { Primer }\end{array}\end{array}$

RT-PCR

$\begin{array}{rr}\text { Cyp19a1 } & \text { F } \\ \text { R }\end{array}$

Actin $\quad$ F

$\mathbf{R}$

qRT-PCR

TCCTTCTCGCTACTTTCAGCCATTTG

ATAAGgGCTGTCTTTCATTCGGGTG

CTGCTCACTGAAGCCCCCC

GGCGTAGCCCTCGTAGATTGG

$\begin{array}{lll}\text { Cyp19a1 } & \text { F } & \text { TCCTTCTCGCTACTTTCAGCCATTTG } \\ & \text { R } & \text { ATAAGGGCTGTCTTTCATTCGGGTG } \\ \text { Rspo1 } & \text { F } & \text { TCCACCTGCCCAGAAGGCTACAC } \\ & \text { R } & \text { TCGTTGCCTTTCTTGAAGCCACAC } \\ \text { Foxl2 } & \text { F } & \text { CCGGCATCTACCAGTACATCATCAGC } \\ & \text { R } & \text { GGGCACCTTGATGAAGCACTCGTT } \\ \text { Dmrt1 } & \text { F } & \text { TGGAAAGCAACAGCCCAACAC } \\ & \text { R } & \text { ATGGCTGGTAAAAACTGCCGTAG } \\ \text { Sox } 9 & \text { F } & \text { GACCATCACCCGCTCGCAG } \\ & \text { R } & \text { GCGTGTACATGGGCCTTTGG } \\ \text { Actin } & \text { F } & \text { CTGCTCACTGAAGCCCCCC } \\ & \text { R } & \text { GGCGTAGCCCTCGTAGATTGG }\end{array}$

